\title{
Correlación y concordancia de tres técnicas de cuantificación de carga viral del VIH disponibles en Colombia
}

\author{
Patricia Olaya ${ }^{1}$, Carlos A. DíazGranados ${ }^{2,3}$ \\ ${ }^{1}$ Centro de Análisis Molecular, Bogotá, D.C., Colombia. \\ ${ }^{2}$ Fundación Universitaria de Ciencias de la Salud (FUCS), Bogotá, D.C., Colombia. \\ ${ }^{3}$ Clínica Jorge Piñeros Corpas, Bogotá, D.C., Colombia.
}

Introducción. La cuantificación del virus de inmunodeficiencia humana (VIH) es una herramienta útil para el manejo de los pacientes infectados. Diferentes técnicas se encuentran disponibles en Colombia para ese propósito.

Objetivo. Comparar los resultados de carga viral obtenidos mediante el uso de tres técnicas de cuantificación del VIH disponibles en Colombia.

Materiales y métodos. Muestras seleccionadas por conveniencia y provenientes de diferentes áreas del país se sometieron a cuantificación del VIH por tres métodos: Versant bDNA $3.0 \AA$ (Bayer), LCx HIV $\AA$ (Abbott) y Amplicor Monitor HIV v1.5 $\AA$ (Roche). Todas las muestras se procesaron en el Centro de Análisis Molecular en Bogotá, Colombia. Los resultados obtenidos con las tres técnicas fueron comparados entre sí mediante regresión lineal. Adicionalmente se determinó la concordancia entre las técnicas calculando el kappa no ponderado, la frecuencia de discordancia según puntos de corte clínicamente relevantes y la frecuencia de diferencias superiores a 0,5 logaritmos.

Resultados. La correlación entre técnicas fue altamente significativa, con un $\mathrm{R}^{2}$ superior a 0,97 . La concordancia también fue sustancial, con kappas no ponderados superiores a 0,7. Sin embargo, las frecuencias de discordancias para puntos de corte clínicamente relevantes y de diferencias superiores a 0,5 logaritmos fueron considerables $(8,8 \%$ a $15,6 \%$ y $19,6 \%$ a $36,3 \%$, respectivamente).

Conclusión. Las tres técnicas para cuantificación de carga viral son adecuadas y muestran un alto nivel de correlación y concordancia. Sin embargo, la variabilidad observada hace necesario comparar la lectura de una técnica con otra lectura realizada por la misma técnica cuando se incorporen dichos resultados en la toma de decisiones clínicas.

Palabras clave: infecciones por VIH, carga viral, reacción en cadena de la polimerasa, diagnóstico, estadísticas no paramétricas, Colombia.

\section{Correlation and concordance of three HIV viral load techniques available in Colombia}

Introduction. Human Immunodeficiency Virus quantitation is a helpful indicator for the management of infected patients. Different technologies are available in Colombia for this purpose.

Objective. To compare the performance of three available technologies for Human Immunodeficiency Virus quantitation in Colombia.

Methods. Samples from different areas of the Country were selected by convenience and underwent Human Immunodeficiency Virus quantitation using three methods: Versant bDNA $3.0 \AA$ (Bayer), LCx HIV ${ }^{\circledR}$ (Abbott) and Amplicor Monitor v1.5 ${ }^{\circledR}$ (Roche). All samples were processed at the Centro de Análisis Molecular in Bogotá, Colombia. Results obtained with the three techniques were compared using linear regression. Additionally, the concordance between techniques was assessed calculating the unweighted kappa, the frequency of discordance according to cut-off points of clinical importance, and the frequency of differences exceeding 0.5 logs.

Results. The correlation between techniques was highly significant, with an $\mathrm{R}^{2}$ higher than 0.97 . The concordance was substantial, with unweighted kappas above 0.7 . Nevertheless, the 
frequency of discordance for cut-off points of clinical importance and the frequency of differences exceeding 0.5 logs were considerable (8.8\%-15.6\% and $19.6 \%-36.3 \%$ respectively).

Conclusion. The three technologies for quantitation of viral load are adequate with high levels of correlation and concordance. However, because of the observed variability we recommend comparing a reading from one technique with another reading from the same technique when incorporating the results into clinical decision making.

Keywords: HIV infection, viral load, polymerase chain reaction, diagnosis, Colombia, nonparametric statistics.

Tanto la cuantificación del ácido ribonucléico (ARN) del virus de la inmunodeficiencia humana (VIH) en plasma, conocida comúnmente como "carga viral", como el recuento de linfocitos CD4 son herramientas útiles para el pronóstico, tratamiento y monitorización de los pacientes infectados con el virus (1-6).

La medición del ARN viral en plasma es parte fundamental de muchos protocolos de manejo de la infección por VIH en diferentes regiones del mundo. Esta ayuda diagnóstica fue recientemente incluida dentro del Plan Obligatorio de Salud (POS) en Colombia (7), facilitando su utilización sistemática en los pacientes colombianos infectados con VIH. Sin embargo, el POS no exige la utilización preferencial de ninguna técnica en particular. En Colombia existen diferentes tecnologías comercialmente disponibles para cuantificación del VIH, que difieren en aspectos tales como su fundamento tecnológico, la cantidad de muestra requerida y los métodos de extracción, amplificación y detección del ácido nucleico. Por la diversidad de programas de atención de pacientes infectados con VIH en Colombia es común que se utilicen técnicas de cuantificación de ARN del VIH diferentes. Con frecuencia un paciente es trasladado de un programa a otro. Adicionalmente, en un mismo programa, la tecnología utilizada puede ser modificada. En estas circunstancias resulta útil y práctico conocer cómo se comparan los resultados de una técnica frente a las demás. La toma de decisiones terapéuticas acertadas y oportunas exige un seguimiento cuidadoso de la infección por VIH

Correspondencia:

Patricia Olaya, Centro de Análisis Molecular, Avenida 13 No 146 -14, Bogotá, Colombia. Telefax: 6154116 patolaya@etb.net.co

Recibido: 20/12/05; aceptado: 25/05/06 por medio de mediciones confiables, precisas y reproducibles de la actividad replicativa del $\mathrm{VIH}$, independientemente de la tecnología utilizada. Con el objetivo de comparar los resultados de carga viral obtenidos mediante el uso de tres técnicas de cuantificación del ARN del VIH disponibles en nuestro medio y en una muestra de pacientes colombianos, se diseñó un estudio de corte transversal en el que todos los especímenes fueron evaluados con las tres técnicas.

\section{Materiales y métodos}

\section{Pacientes y muestras}

Se calculó un tamaño de muestra para correlación asumiendo un alfa de 0,05 , un beta de 0,2 , una correlación basal de 0,95 y una correlación esperada de 0,975. De acuerdo con lo anterior, el tamaño de muestra necesario para el estudio se estimó en 87 pacientes (NCSS \& PASS software versión 5.1, Kaysville, Utah). Se seleccionaron 102 muestras por conveniencia para ser evaluadas por triplicado. La mitad de las muestras se obtuvieron de pacientes con diagnóstico de VIH manejados en el programa de VIH-SIDA de la Corporación Saludcoop Cundinamarca, Colombia. La otra mitad se seleccionó de muestras de otros programas del país que solicitaran medición de carga viral al Centro de Análisis Molecular en Bogotá, Colombia. A todas las muestras se les realizó cuantificación del ARN del VIH por tres métodos: Versant bDNA 3.0 $\AA$ (Bayer), LCx HIV (A) (Abbott) y Amplicor Monitor HIV v1.5 $\AA$ (Roche). Todas las muestras se manejaron, procesaron y analizaron en el Centro de Análisis Molecular y fueron interpretadas por un único observador (P.O.).

Para la toma de las muestras se emplearon dos tubos PPT®) (tubos al vacío con EDTA y gel libres de ADNsas y ARNsas, $B D{ }^{\circledR}$ ) por cada paciente. 
Las dos muestras se tomaron con aguja múltiple, por punción venosa, una inmediatamente después de la otra y fueron centrifugadas simultáneamente dentro de las dos horas posteriores a la extracción a 2.500 RPM $(1.300 \times \mathrm{g})$ durante 15 minutos y a temperatura ambiente. El procedimiento y las instrucciones para el manejo y transporte de las muestras se incluyeron en un protocolo que se distribuyó en todos los puntos de toma de muestras; el transporte se realizó a temperatura ambiente por mensajería rápida y las muestras fueron recibidas dentro de las 24 horas siguientes a la toma. De cada muestra se hicieron tres alícuotas y se almacenaron a $-70{ }^{\circ} \mathrm{C}$ hasta su procesamiento, el cual se realizó con los tres métodos en un tiempo máximo de siete días.

\section{Métodos de laboratorio}

La determinación de la carga viral se ha descrito en detalle en otras publicaciones (8-10). Las características principales de cada técnica se describen brevemente a continuación.

Versant bDNA 3.0 ( $)$ : emplea 1,0 mL de plasma y se basa en la tecnología de amplificación de señal según la cual el ARN del VIH se somete a hibridización con una serie de sondas de oligonucleótidos complementarios a regiones altamente conservadas del gen pol del $\mathrm{VIH}-1$. La prueba tiene un rango dinámico de 50 a 500.000 copias de ARN viral por $\mathrm{mL}$ (8).

LCx HIV ®: es una técnica competitiva de reacción en cadena de la polimerasa (PCR) que utiliza iniciadores que se unen a una región altamente conservada del gen pol-integrasa del VIH. La detección y cuantificación se realiza de manera automática con un sistema de inmunoensayo de micropartículas. Ofrece dos posibilidades para el procesamiento de las muestras: un protocolo con $1,0 \mathrm{~mL}$ de muestra con un rango dinámico entre 50 y un millón de copias de ARN viral por $\mathrm{mL}$, y un protocolo con $0,2 \mathrm{~mL}$ de muestra, con un rango dinámico entre 178 y cinco millones de copias de ARN viral por $\mathrm{mL}$. Para este estudio se empleó el protocolo con 1,0 mL de muestra (9).

Amplicor Monitor HIV v1.5 ®: emplea una técnica de PCR que amplifica la región p24 del gen gag del VIH. Existen dos procedimientos, el ultrasensible y el estándar. Los rangos dinámicos son de 50 a 75.000 copias $/ \mathrm{mL}$ y de 400 a 750.000 copias $/ \mathrm{mL}$, respectivamente (10). Para decidir qué procedimiento de Amplicor Monitor HIV v1.5 ${ }^{\circledR}$ utilizar se empleó el resultado de carga viral de cualquiera de las otras dos técnicas.

\section{Análisis}

Las tres técnicas se compararon mediante regresión lineal, correlacionando entre sí los logaritmos del resultado del número de copias de las tres técnicas. Se calculó para cada comparación el coeficiente de correlación de Pearson ( $r$ ), el $R^{2}$ y el valor de $p$. Adicionalmente, se determinó la concordancia entre técnicas categorizando el número de copias según puntos de corte críticos para la toma de decisiones clínicas (menor a 400 copias, entre 400 y 100.000 copias y mayor a 100.000 copias [1,11-13]), y se calculó el nivel kappa no ponderado para cada comparación. El grado de concordancia para cada comparación se clasificó usando la jerarquía de Landis y Koch (14). Se determinó la frecuencia con la cual las técnicas generarían conductas diagnósticas o terapéuticas diferentes en un mismo momento en el tiempo mediante el recuento de circunstancias en las cuales se reportara para una técnica una carga viral menor de 400 copias y para la otra una carga viral mayor o igual a 400 copias, o para una técnica una carga viral menor o igual a 100.000 copias y para la otra una carga viral mayor a 100.000 copias. Finalmente, se calculó la diferencia del logaritmo de la carga viral entre una técnica y otra, y se determinó la frecuencia con la que dicha diferencia superó los 0,5 logaritmos. Lo anterior permite estimar con qué frecuencia el clínico podría considerar equivocadamente que ha ocurrido un cambio significativo en la carga viral del paciente durante el seguimiento (15). Se utilizó el software SAS versión 8.2 (SAS Institute) para llevar a cabo los análisis estadísticos. Se consideraron significativos valores de $p$ menores a 0,05.

\section{Aspectos éticos}

El estudio fue aprobado por los Comités de Investigación y Ética de las Empresas Promotoras de Salud (EPS) Saludcoop y Compensar. Las muestras pertenecían a pacientes para quienes 
el médico tratante había ordenado la cuantificación de la carga viral por alguna de las técnicas incluidas en el estudio y, por lo tanto, su obtención no requirió de procedimientos ni riesgos adicionales para los pacientes; la realización del estudio no causó demoras ni alteración en los resultados y no afectó el manejo clínico de los pacientes. La confidencialidad y privacidad de los pacientes se protegió durante la recolección y transporte de las muestras y durante la interpretación y reporte de los resultados.

\section{Resultados}

El cuadro 1 muestra la frecuencia con la cual los resultados fueron discordantes para puntos de corte clínicamente relevantes y la frecuencia con que la diferencia de cargas virales superó los 0,5 logaritmos. Los cuadros 2, 3 y 4 muestran el número de casos concordantes y discordantes para cada categoría de carga viral entre Amplicor Monitor HIV y bDNA, Amplicor Monitor HIV y LCx y bDNA y LCx, respectivamente.

\section{Comparación de Amplicor Monitor HIV v1.5 y bDNA}

La correlación de las pruebas fue altamente significativa $\left(r=0,953, R^{2}=0,976, p<0,001\right.$, figura 1). La concordancia entre las pruebas fue casi perfecta (kappa $=0,85, \mathrm{IC} 95 \%$ entre 0,75 y 0,94, valor de $p<0,001)$. La diferencia promedio entre el logaritmo del número de copias de la carga viral según Amplicor Monitor HIV v1.5 y bDNA fue de 0,14+/- 0,3 logaritmos (IC95\% entre 0,08 y 0,2).

\section{Comparación de Amplicor Monitor HIV v1.5 y $L C x$}

La correlación de las pruebas fue altamente significativa $\left(r=0,946, R^{2}=0,973, p<0,001\right.$, figura 2). La concordancia entre las pruebas fue sustancial $(\mathrm{kappa}=0,74, \mathrm{IC} 95 \%$ entre 0,63 y $0,85$, valor de $p<0,001)$. La diferencia promedio entre el logaritmo del número de copias de la carga viral según LCx y Amplicor Monitor HIV v1.5 fue de $0,17+/-0,38$ logaritmos (IC95\% entre 0,09 y $0,24)$.

\section{Comparación de bDNA y LCx}

La correlación de las pruebas fue altamente significativa $\left(r=0,95, R^{2}=0,975, p<0,001\right.$,
Cuadro 1. Frecuencia de discordancia en puntos de corte relevantes y de diferencias superiores a $0,5 \mathrm{log}$. Versant bDNA 3.0, LCx HIV y Amplicor Monitor HIV v1.5

\begin{tabular}{lcc}
\hline $\begin{array}{l}\text { Técnicas } \\
\text { comparadas }\end{array}$ & $\begin{array}{c}\text { Discordancia puntos } \\
\text { de corte relevantes }\end{array}$ & $\begin{array}{c}\text { Diferencias } \\
\text { superiores } \\
\mathbf{a} \mathbf{0 , 5} \text { log } \\
\mathbf{n ~ ( \% )}\end{array}$ \\
\hline LCx - bDNA & $15(14,7 \%)$ & $37(36,3 \%)$ \\
LCx - Monitor v1.5 & $16(15,6 \%)$ & $22(21,6 \%)$ \\
Monitor v1.5 - bDNA & $9(8,8 \%)$ & $20(19,6 \%)$ \\
\hline
\end{tabular}

Cuadro 2. Concordancia y discordancia de Amplicor Monitor HIV v1.5 y Versant bDNA 3.0 para las diferentes categorías de carga viral de importancia clínica. Los contenidos de cada casilla se expresan en números absolutos (número total de observaciones con cada técnica $=102$ ).

\begin{tabular}{ccccc}
\hline & \multicolumn{4}{c}{ Amplicor Monitor HIV } \\
\cline { 2 - 5 } & & $\begin{array}{c}<\mathbf{4 0 0} \\
\text { copias }\end{array}$ & $\begin{array}{c}\mathbf{4 0 0 - 1 0 0 . 0 0 0} \\
\text { copias }\end{array}$ & $\begin{array}{c}\mathbf{8 1 0 0 . 0 0 0} \\
\text { copias }\end{array}$ \\
\hline bDNA & $\begin{array}{c}\text { copias } \\
\text { cop-100.000 } \\
\text { copias } \\
100.000\end{array}$ & 49 & 2 & 0 \\
copias & 0 & 36 & 2 \\
\hline
\end{tabular}

Cuadro 3. Concordancia y discordancia de Amplicor Monitor HIV v1.5 y LCx HIV para las diferentes categorías de importancia clínica. Los contenidos de cada casilla se expresan en números absolutos (número total de observaciones con cada técnica $=102$ ).

\begin{tabular}{|c|c|c|c|c|}
\hline & & \multicolumn{3}{|c|}{ Amplicor Monitor HIV } \\
\hline & & $\begin{array}{l}<400 \\
\text { copias }\end{array}$ & $\begin{array}{c}400-100.000 \\
\text { copias }\end{array}$ & $\begin{array}{c}>100.000 \\
\text { copias }\end{array}$ \\
\hline \multirow{3}{*}{ LCx } & $\begin{array}{c}<400 \\
\text { copias }\end{array}$ & 47 & 4 & 0 \\
\hline & $\begin{array}{c}400-100.000 \\
\text { copias }\end{array}$ & 2 & 28 & 10 \\
\hline & $\begin{array}{c}>100.000 \\
\text { copias }\end{array}$ & 0 & 0 & 11 \\
\hline
\end{tabular}

figura 3). La concordancia entre las pruebas fue sustancial (kappa $=0,76$, IC95\% entre 0,65 y $0,87$, valor de $p<0,001)$. La diferencia promedio entre el logaritmo del número de copias de la carga viral según LCx y bDNA fue de 0,31+/- 0,39 logaritmos (IC95\% entre 0,22 y 0,38). 
Cuadro 4. Concordancia y discordancia de Versant bDNA 3.0 y LCx HIV para las diferentes categorías de importancia clínica. Los contenidos de cada casilla se expresan en números absolutos (número total de observaciones con cada técnica = 102).

\begin{tabular}{ccccc}
\hline & \multicolumn{4}{c}{ LCX } \\
\cline { 3 - 5 } & & $\begin{array}{c}<00 \\
\text { copias }\end{array}$ & $\begin{array}{c}\mathbf{4 0 0 - 1 0 0 . 0 0 0} \\
\text { copias }\end{array}$ & $\begin{array}{c}>100.000 \\
\text { copias }\end{array}$ \\
\hline bDNA & $\begin{array}{c}\text { copias } \\
\text { con-100.000 }\end{array}$ & 48 & 1 & 0 \\
copias & 3 & 29 & 0 \\
& & & & \\
& 100.000 & 0 & 11 & 10 \\
copias & & & \\
\hline
\end{tabular}

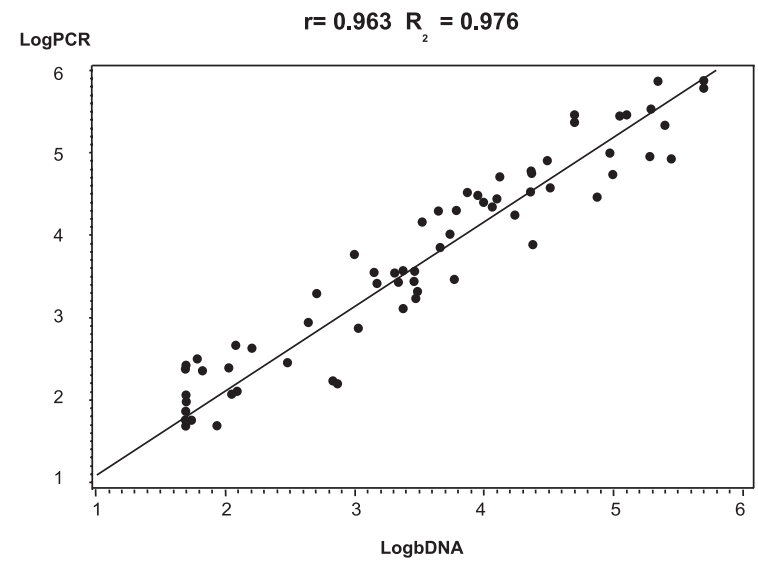

Figura 1. Correlación entre logaritmos de carga viral $\left(\log _{10}\right.$ copias $\left.A R N / m L\right)$ con técnicas Versant bDNA 3.0 y Amplicor Monitor HIV v1.5.

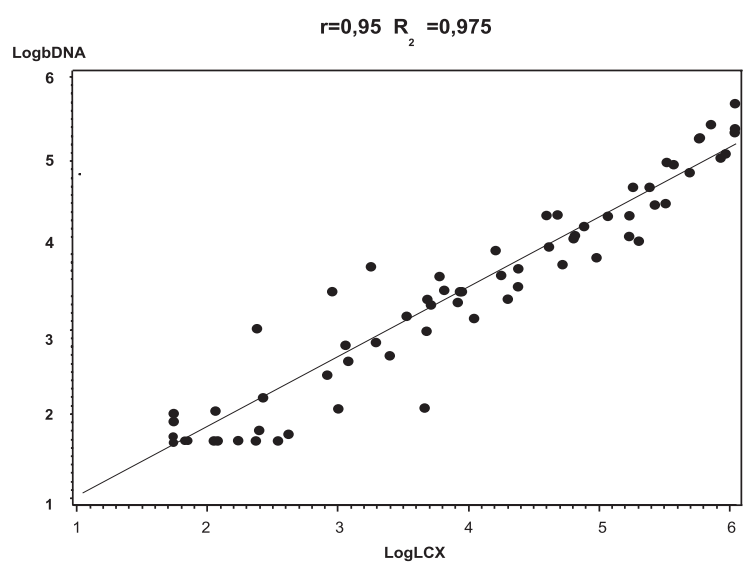

Figura 2. Correlación entre logaritmos de carga viral $\left(\log _{10}\right.$ copias $\left.A R N / m L\right)$ con técnicas Versant bDNA 3.0 y LCX HIV.

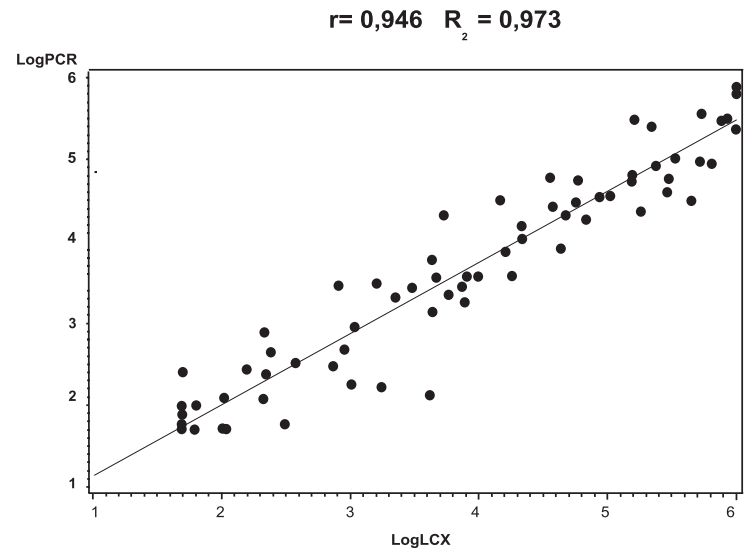

Figura 3. Correlación entre logaritmos de carga viral $\left(\log _{10}\right.$ copias $\left.A R N / m L\right)$ con técnicas Amplicor Monitor HIV v1.5 y LCx HIV.

\section{Discusión}

El presente estudio compara lo que una y otra técnicas de cuantificación de carga viral "observan", independientemente de lo que esté sucediendo con el paciente. Resulta de particular interés evaluar pacientes en diferentes fases de la infección, para quienes el rango de carga viral incluya cargas virales bajas, intermedias y altas. Nuestro estudio muestra un excelente nivel de correlación entre técnicas. El coeficiente de correlación para todas las comparaciones fue mayor de 0,94 con un $\mathrm{R}^{2}$ mayor de 0,97 , lo cual significa que la variabilidad de una técnica puede explicarse casi en su totalidad por la variabilidad de la otra. Las pruebas mostraron también excelente concordancia, con kappas no ponderados por encima de 0,7. La correlación y concordancia fue adecuada con los niveles bajos de los rangos dinámicos de las pruebas utilizadas, sugiriendo una sensibilidad similar para las tres técnicas. Estudios previos han mostrado excelente correlación y concordancia entre Amplicor Monitor HIV v1.5 y Versant bDNA 3.0 (16-19). Similarmente, aunque algunos reportes difieren (20), la mayoría de estudios han mostrado excelente correlación y concordancia entre LCx HIV y las otras técnicas $(16,17,21)$. Este estudio no incluyó ningún tipo de análisis filogenético para la clasificación de los subtipos de VIH de los pacientes. Por lo tanto la repercusión de la 
presencia de diferentes subtipos de VIH en los resultados es desconocida. Sin embargo, aunque limitados, los estudios filogenéticos realizados en Colombia indican un claro predominio del subtipo B en nuestra población $(22,23)$.

En promedio, las lecturas realizadas con la técnica LCx HIV resultaron superiores a las de Amplicor Monitor HIV v1.5 y Versant bDNA $3.0{ }^{\circledR}$. Esto concuerda con lo descrito en reportes previos $(13,16)$. Las lecturas de Versant bDNA 3.0 resultaron levemente inferiores a las de Amplicor Monitor HIV 1.5. Lo anterior genera el interrogante de si las decisiones de manejo serían diferentes cuando se utiliza indistintamente una u otra técnica. Un $15,6 \%$ de las pruebas resultó discordante en puntos de corte críticos cuando se comparó LCx HIV con Amplicor Monitor HIV v1.5. Similarmente, un $14,7 \%$ y un $8,8 \%$ de las pruebas resultó discordante en los puntos de corte críticos cuando se comparó LCx HIV con Versant bDNA 3.0 y Versant bDNA 3.0 con Amplicor Monitor HIV v1.5. Además, la diferencia del logaritmo de la carga viral de una técnica a otra fue superior a 0,5 logaritmos en un número importante de casos. Lo anterior permite afirmar que si bien las tres técnicas tienen una excelente correlación y concordancia estadística, las decisiones clínicas se afectarían frecuentemente si las técnicas se intercambian indistintamente. Los pacientes infectados con VIH frecuentemente son monitorizados de manera seriada mediante la medición de la carga viral. Suponiendo que después de unos meses de seguimiento no ha ocurrido ningún cambio en la cantidad "real" de virus de un paciente, la lectura de la carga viral tampoco debería cambiar, y el resultado reportado debería ser similar al previo. Sin embargo, nuestro estudio muestra que si se usan las técnicas de manera indistinta, con frecuencia el clínico podría erróneamente suponer que ha ocurrido un cambio significativo en la carga viral, cuando en realidad el cambio se explicaría completamente por una modificación de la técnica. Por ello es recomendable que al tomar decisiones terapéuticas se comparen los resultados de una técnica con resultados previos o duplicados de la misma técnica. Específicamente, si un programa de atención de pacientes con VIH va a modificar la técnica empleada para determinar la carga viral, debe obtenerse de cada paciente un nivel basal de carga viral con la técnica a instaurar, con el fin de hacer comparaciones adecuadas durante el seguimiento clínico y terapéutico.

Nuestro estudio indica que las tres técnicas de cuantificación de carga viral son adecuadas, ya que muestran un alto nivel de correlación y concordancia, lo que permite la utilización clínica de cualquiera de ellas. Sin embargo, la variabilidad observada, aunque pequeña, hace necesaria la utilización del mismo método de cuantificación de la carga viral para la toma de decisiones clínicas en el seguimiento de los pacientes a través del tiempo o para establecer el nuevo valor de base con la técnica a instaurar.

\section{Agradecimientos}

Los autores agradecen a Juan A. Benavides, Director del Centro de Investigaciones de la EPS Saludcoop y a la bacterióloga Carmen Cecilia Trujillo, Gerente del Laboratorio Clínico de la IPS Compensar.

\section{Conflicto de Intereses}

Este estudio fue financiado de manera irrestricta por laboratorios Abbott.

El patrocinador del estudio no tuvo ningún papel en el diseño, conducción, o conclusión del estudio. El Centro de Análisis Molecular tiene relaciones comerciales con los laboratorios Abbott, Roche, y Bayer. El presente estudio no modificó la relación comercial existente previa a su desarrollo. Carlos A. DiazGranados no tiene ningún tipo de relación comercial con el patrocinador del estudio ni con otro de los laboratorios mencionados.

\section{Referencias}

1. Phillips A, CASCADE Collaboration. Short-term risk of AIDS according to current CD4 cell count and viral load in antiretroviral drug-naïve individuals and those treated in the monotherapy era. AIDS 2004;18:51-8.

2. O'Brien WA, Hartigan PM, Martin D, Esinhart J, Hill A, Benoit S, et al. Changes in plasma HIV-1 RNA and CD4+ lymphocyte counts and the risk of progression to AIDS. N Engl J Med 1996;334:426-31.

3. Katzenstein DA, Hammer SM, Hughes MD, Gundacker H, Jackson JB, Fiscus S, et al. The relation of virologic and immunologic markers to clinical 
outcomes after nucleoside therapy in HIV-Infected adults with 200 to 500 CD4 cells per cubic millimeter. N Engl J Med 1996;335:1091-8.

4. Philips AN, Eron J, Bartlett J, Kuritzkes DR, Johnson VA, Gilbert C, et al. Correspondence between the effect of zidovudine plus lamivudine on plasma HIV level/CD4 lymphocyte count and the incidence of clinical disease in infected individuals. AIDS 1997;11:169-75.

5. Marschner IC, Collier AC, Coombs RW, D'Aquila RT, DeGruttola V, Fischl MA, et al. Use of changes in plasma levels of Human Immunodeficiency Virus Type 1 RNA to assess the clinical benefit of antiretroviral therapy. J Infect Dis 1998;177:40-7.

6. Delta Coordinating Committee and Virology Group. An evaluation of HIV RNA and CD4 cell count as surrogates for clinical outcome. AIDS 1999;13:565-73.

7. Ministerio de la Protección Social, República de Colombia. Acuerdo CNSSS 254 de 2003. [Consultado: diciembre 1de 2005]. Disponible en: http:// www.minproteccionsocial.gov.co/MseContent/images/ news/DocNewsNo453201.pdf.

8. Collins ML, Irvine B, Tyner D, Fine E, Zayati C, Chang $\mathbf{C}$, et al. A branched DNA signal amplification assay for quantification of nucleic acid targets below 100 molecules/ml. Nucl Acids Res 1997;25:2979-84.

9. Johanson J, Abravaya K, Caminiti W, Erickson D, Flanders R, Leckie G, et al. A new ultrasensitive assay for quantitation of HIV-1 RNA in plasma. J Virol Methods 2001;95:81-92.

10. Sun R, Ku J, Jayakar H, Kuo JC, Brambilla D, Herman S, et al. Ultrasensitive reverse transcriptionPCR assay for quantitation of human immunodeficiency virus type 1 RNA in plasma. J Clin Microbiol 1998;36: 2964-9.

11. DeJesus E, McCarty D, Farthing CF, Shortino DD, Grinsztejn B, Thomas BA, et al. Once-daily versus twice-daily lamivudine, in combination with zidovudine and efavirenz, for the treatment of antiretroviral-naive adults with HIV Infection: a randomized equivalence trial. Clin Infect Dis 2004;39:411-8.

12. Walmsley S, Bernstein B, King M, Arribas J, Beall G, Ruane $\mathbf{P}$, et al. Lopinavir-ritonavir versus nelfinavir for the initial treatment of HIV infection. N Engl J Med 2002;346:2039-46.

13. Saag MS, Cahn P, Raffi F, Wolff M, Pearce D, Molina JM, et al. Efficacy and safety of emtricitabine vs stavudine in combination therapy in antiretroviral-naive patients: a randomized trial. JAMA 2004;292:180-9.

14. Landis JR, Koch GG. The measurement of observer agreement for categorical data. Biometrics 1977;33:159-74.

15. US Department of Health and Human Services (DHHS). Guidelines for the use of antiretroviral agents in HIV-1-infected adults and adolescents. [Consultado: julio 5 de 2005]. Disponible en: http://aidsinfo.nih.gov/ guidelines/adult/AA_100605.pdf.

16. de Mendoza C, Alcami J, Sainz M, Folgueira D, Soriano V. Evaluation of the Abbott LCx quantitative assay for measurement of human immunodeficiency virus RNA in plasma. J Clin Microbiol 2002; 40:151821.

17. Swanson P, Soriano V, Devare SG, Hackett J Jr. Comparative performance of three viral load assays on human immunodeficiency virus type 1 (HIV-1) isolates representing group $M$ (subtypes $A$ to $G$ ) and group O: LCx HIV RNA quantitative, AMPLICOR HIV-1 MONITOR version 1.5, and Quantiplex HIV-1 RNA version 3.0. J Clin Microbiol 2001;39:862-70.

18. Zanchetta N, Nardi G, Tocalli L, Drago L, Bossi C, Pulvirenti FR, et al. Evaluation of the Abbott LCx HIV1 RNA quantitative, a new assay for quantitative determination of human immunodeficiency virus type 1 RNA. J Clin Microbiol 2000;38:3882-6.

19. Katsoulidou A, Papachristou E, Petrodaskalaki M, Sypsa V, Anastassopoulou CG, Gargalianos P, et al. Comparison of three current viral load assays for the quantitation of human immunodeficiency virus type 1 RNA in plasma. J Virol Methods 2004;121:93-9.

20. Swanson P, Harris BJ, Holzmayer V, Devare SG, Schochetman G, Hackett J Jr. Quantification of HIV1 group M (subtypes AG) and group O by the LCx HIV RNA quantitative assay. J Virol Methods 2000;89:97108.

21. Plantier JC, Gueudin M, Damond F, Braun J, Mauclere P, Simon F. Plasma RNA quantification and HIV-1 divergent strains. J Acquir Immune Defic Syndr 2003;33:1-7.

22. Navas MC, Letourneur F, Gomas E, Boshell J, Saragosti S. Analysis of the V3 loop sequences from 12 HIV type 1-infected patients from Colombia, South America. AIDS Res Hum Retroviruses 1999;15:11414.

23. Carrion G, Hierholzer J, Montano S, Alava A, Perez J, Guevara A, et al. Circulating recombinant form CRF02_AG in South America. AIDS Res Hum Retroviruses 2003;19:329-32. 